\title{
Construction of evaluation system based on Collaborative Innovation Perspective on the function national of high-tech zones
}

\author{
Wangwei $^{1}$ Xiongshan $^{2}$ Zhangyong $^{3}$ \\ Institute of Economics \& Management, Sichuan University of Science and Engineering \\ Sichuan Zigong 643000, China \\ a369946541@qq.com
}

Keywords: AHP, National High-tech Zone, Collaborative Innovation.

\begin{abstract}
Dynamic reasonable and objective evaluation for the development status of high-tech zone will grasp the development trend of high-tech zone accurately and have a very positive effect on promoting adjustment policies. Through the use of AHP, building a high-tech zone evaluation system from the perspective of collaborative innovation, contains a set of independent innovation capabilities, industry consolidation upgrade feature, open competitive functions and collaborative ecological demonstration of innovative features, provides theoretical and intellectual support.
\end{abstract}

\section{Introduction}

High-tech Zone is a product formed after China's reform and opening up. Since 1988, China begin to build high-tech zone, after twenty years' energetic sustain and development, high-tech zones both in quantity and in quality have made significant achievements domestic scholars have launched a high-tech zone theoretical and empirical research boom.

After the "first venture", "second pioneering" stage, China's high-tech zone has gradually become the main carrier of regional economic development. With the development, improving the function of high-tech zone system has also become a top priority. In recent years, research on collaborative innovation ideas is growing. Bring the collaborative innovative ideas into the category of high-tech zone function is the general trend of the times. Therefore, it is not only very important to build a scientific, rational and objective evaluation index system for the high-tech zone from the perspective of collaborative innovation, but also provide theoretical and intellectual support for all levels of government in the practice of high-tech zone development.

\section{Literature summaries}

High-tech Zone, as the mainstay of regional economic development, whether abroad or in the country has attracted the attention of a large number of scholars. High-tech Zone Evaluation Index System also has become the focus of research scholars at home and abroad.

As early as 1985, the American scholar Rogers and Larsen, study the process of Silicon Valley growth and start with qualitative analysis method, and explains its "Strengthening economic effect" conditions [1]. In 1987, Makecki designed an evaluation system includes eight indicators, assessed the ability of high-tech zone development from science and technology talent and venture capital mobility and other aspects [2]. In 1988, Malechi and Nijkamp designed a evaluation system on the regional condition, including research institutions and high-quality skilled labor, etc. [3]. In 1999, Silicon Valley Internet companies have designed a third-level evaluation index system study Silicon Valley's annual comprehensive cases; the system includes five-level indicators, 15 secondary indicators and 66 third-level indicators. In 2004, ShuE Mei and others designed an evaluation index system, included technological innovation level, incubators construction level, high-tech industrial development level, economic and social development level and international level, investigated the high-tech zone development level [4]. In 2009, Jiaxiang Chen established an evaluation system form innovation capacity and performance innovative two aspects, evaluated 53 national high-tech zones, and found there was some function alienation [5]. In 2011, Shuhua Hu built a multi-level fuzzy 
comprehensive evaluation model, evaluated the competitive of National High-tech Zone from the support, innovation, contribute and international competitiveness four dimensions [6].

Research on collaborative innovation, scholars had achieved significant results. In 1997, Zhang proposed general pattern about technical, organizational and cultural collaborative innovation [7]. In 2008, Gang Zheng proposed C3IR five-stage comprehensive collaborative innovation process model [8]. In 2009, Decheng Fan established a index system about Production, Education \& Research collaborative innovation, evaluated Chinese technological innovation [9]; Minghua Jin establish a collaborative innovation matter-element model to assess innovative risk, from the perspective of qualitative and quantitative evaluated the collaborative innovation risk level of business and the customer [10]. 2013, Guangjun Ou and others evaluated collaborative innovation ability of high-tech zones from the perspective of industrial clusters, and found that most parks have drawback in the environmental cofactors, endogenous cofactors and collaborative motivation factor [11] .

\section{Theory primaries of evaluation}

Determining index system is the focus of the entire high-tech zone evaluation, however, it is a very complex task to establish a comprehensive, scientific and rational evaluation system, which can reflect the high-tech zone functions. Based on the systematic analysis for high-tech zones, combined with the research results of many experts and scholars, and considered the availability of index data.

\section{Screening Evaluation by Expert}

Through theoretical primaries, initially establish a high-tech zone function evaluation system. However, to build a scientific and reasonable indicator system, theoretical primaries is not enough, we must execute expert screening.

\section{Using the analytic hierarchy process (AHP) to determine index weight}

Track the analytic hierarchy process (AHP):analytic hierarchy process (AHP) is a combination of quantitative and qualitative research method the characteristic of this method is a complex decision problem in nature, after analyzing the influencing factors and inherent relations, building a hierarchy structure model, and then taking advantage of less quantitative information, the decisions of mathematical thinking process, for solving the multi-objective, guidelines, or no structural characteristics of complex problems, which can provide a simple decision method. National hi-tech zone function evaluation index system is a set of hierarchical structure model, which involves index has both qualitative and quantitative .therefore, using the analytic hierarchy process to the construction of index system is very good to solve the above two problems.

The steps of the AHP method are:

(1)Analytic hierarchy structure model is established

Through this process, the complex problem that we will be solved is decomposed into the component of each element, according to the relationship between elements and subordinate relations form different levels, namely the target layer, criterion layer and scheme, hierarchy analysis model of the structure.

(2) Construct judgment matrix

Judgment matrix is two elements in the same level two relatively important to calculate the important basis. All aspects of a certain number of expert groups, to eventually form the same level of each index in the index system of the two compare and assignment, weighted, and finally form a judgment matrix. 
Table1 Indicators relative importance evaluation level and assignment

\begin{tabular}{|l|l|l|}
\hline Number & Importance level & Assignment \\
\hline 1 & i as important as $\mathrm{j}$ & 1 \\
\hline 2 & i is slightly more important than $\mathrm{j}$ & 3 \\
\hline 3 & i is significantly more important than $\mathrm{j}$ & 5 \\
\hline 4 & i is strongly more important than $\mathrm{j}$ & 7 \\
\hline 5 & i is extreme more important than $\mathrm{j}$ & 9 \\
\hline 6 & i is slightly less important than $\mathrm{j}$ & $1 / 3$ \\
\hline 7 & i is obviously less important than $\mathrm{j}$ & $1 / 5$ \\
\hline 8 & i is highly less important than $\mathrm{j}$ & $1 / 7$ \\
\hline 9 & i is extremely less important than $\mathrm{j}$ & $1 / 9$ \\
\hline
\end{tabular}

Note: Assignment $=\{2,4,6,8,1 / 2,1 / 4,1 / 6,1 / 8\}$ indicates the importance of grades between assignments $=\{1,3,5,7,9,1 / 3,1 / 5,1 / 7,1 / 9\}$.

To construct the judgment matrix A, as follows:

If $0.25<Z_{i j}-Z_{i k} \leqslant 0.5, Z_{i j}$ is slightly more important than $Z_{i k}$, saaty scale take 3;

If $0.75<Z_{i j}-Z_{i k} \leqslant 1, Z_{i j}$ is significantly more important than $Z_{i k}$, saaty scale take 5;

If $1.25<Z_{i j}-Z_{i k} \leqslant 1.5, Z_{i j}$ is strongly important than $Z_{i k}$, saaty scale take 7;

If $1.75<Z_{i j}-Z_{i k} \leqslant 2, Z_{i j}$ is extremely important than $Z_{i k}$, saaty scale take 9;

If the difference between the two scales, the scales take saaty 2,4,6,8. According to this principle, the structure of the judgment matrix A, and calculate the final weights.

(3) Single sort and consistency inspection level

Hierarchical single sort through calculating the maximum characteristic value of judgment matrix, steps are as follows:

(1) Calculate the product of judgment matrix' s each row elements $M_{i}$

$$
M_{i}=\prod_{j=1}^{n} c_{i j} \quad \mathrm{i}=1,2, \cdots, \mathrm{n} \quad \text { Formula } 1
$$

(2) Calculate the n-th root of $M_{i} \quad \overline{W_{i}} \quad \overline{W_{i}}=\sqrt[n]{M_{i}}$

(3) The vector $\bar{W}=\left[\overline{W_{1}}, \overline{W_{2}}, \cdots, \overline{W_{n}}\right] \mathrm{T}$ is normalized handled

$W_{i}=\frac{\overline{W_{i}}}{\sum_{j=1}^{n} \overline{W_{i}}}$

Formula 2

So $W=\left[W_{1}, W_{2}, \cdots, W_{n}\right] \mathrm{T}$ is the required eigenvectors.

(4) Calculate the maximum Eigen value of judgment matrix

$\lambda_{\max }=\sum_{i=1}^{n} \frac{(A W)_{i}}{n W_{i}}$

Formula 3

$(A W)_{i}$ represents the i-th element of the vector.

Calculate constructed Judgment matrix maximum Eigen value $\lambda$ max and corresponding feature vector $\bar{W}$, using consistency index ( $C I$ ), random consistency index ( $R I$ ) and consistency ratio ( $C R$ ) do consistency test. If $C R<0.1$, then consistency, the feature vector (normalized) is the weight vector $W$ : If $C R>0.1$, then no consistency, we should reconstructed matrices.

Among them:

$$
C I=\frac{\lambda \max -n}{n-1} \text { Formula } 4
$$




$$
C R=\frac{C I}{R I} \quad \text { Formula } 5
$$

When CR $<0.1$, the judgment matrix has satisfactory consistency.

Table 2 RI value of Average random consistency index

\begin{tabular}{l|l|l|l|l|l|l|l|l}
\hline 1 & 2 & 3 & 4 & 5 & 6 & 7 & 8 & 9 \\
\hline 0.00 & 0.00 & 0.58 & 0.90 & 1.12 & 1.24 & 1.32 & 1.41 & 1.45 \\
\hline
\end{tabular}

(4) The level of total sorts and consistency check

In turn along the recursive structure calculation one by one down the class time, we can calculate the bottom factors relative to the top (total goal) the relative importance or value in the relative advantages and disadvantages, namely hierarchy total ordering. The consistency check is from top to bottom.

Determine the index weights:Using the analytic hierarchy software, calculate independent innovation function, industry consolidation upgrade feature, opening competition function, ecological demonstration function, collaborative innovation function five-level indicator judgment matrix, as follows:

$\mathrm{A}=\left[\begin{array}{ccccc}1 & 2 & 5 & 3 & 1 \\ \frac{1}{2} & 1 & 3 & 2 & \frac{1}{2} \\ \frac{1}{5} & \frac{1}{3} & 1 & \frac{1}{3} & \frac{1}{2} \\ \frac{1}{3} & \frac{1}{2} & 3 & 1 & \frac{1}{4} \\ 1 & 2 & 2 & 4 & 1\end{array}\right]_{5 \text { multiplication open square }}^{\longrightarrow}\left[\begin{array}{c}1.76 \\ 1.07 \\ 0.47 \\ 0.74 \\ 1.59\end{array}\right] \stackrel{\text { The same treatment }}{\longrightarrow} W=\left[\begin{array}{l}0.31 \\ 0.19 \\ 0.08 \\ 0.13 \\ 0.28\end{array}\right]$

So $A W=\left[\begin{array}{lllll}1.76 & 0.99 & 0.39 & 0.64 & 1.65\end{array}\right]$

Calculated according to the formula 3

$$
\lambda \text { max }=\frac{1}{5}\left[\frac{1.76}{0.31}+\frac{0.99}{0.19}+\frac{0.39}{0.08}+\frac{0.64}{0.13}+\frac{1.65}{0.28}\right]=5.32
$$

Calculated according to the formula 4

$$
C I=\frac{5.32-5}{5-1}=0.08
$$

$R I=1.12$

Calculated according to the formula 5

$$
C R=\frac{C I}{R I}=\frac{0.08}{1.12}=0.07<0.1
$$

Therefore, the judgment matrix has satisfactory consistency, weight vectors suitable for decision analysis.

Similarly, calculate secondary level index weight, the final outcome as shown in Table 3. 
Table 3 Indicators weights of national high-tech zones evaluation index system

\begin{tabular}{|c|c|c|c|c|c|}
\hline $\begin{array}{l}\text { Variable } \\
\text { Symbol }\end{array}$ & B1 & B2 & B3 & B4 & B5 \\
\hline Weights & 0.3366 & 0.1848 & 0.0693 & 0.1125 & 0.2968 \\
\hline $\begin{array}{l}\text { Variable } \\
\text { Symbol }\end{array}$ & $\begin{array}{l}\text { Weigh } \\
\text { ts }\end{array}$ & $\begin{array}{l}\text { Variable } \\
\text { Symbol }\end{array}$ & Weights & $\begin{array}{l}\text { Variable } \\
\text { Symbol }\end{array}$ & Weights \\
\hline $\mathrm{C} 1$ & 0.1587 & C15 & 0.1090 & C29 & 0.1278 \\
\hline C2 & 0.0667 & C16 & 0.1549 & C30 & 0.0799 \\
\hline C3 & 0.0716 & C17 & 0.0985 & C31 & 0.1464 \\
\hline $\mathrm{C} 4$ & 0.1666 & C18 & 0.1294 & C32 & 0.1327 \\
\hline C5 & 0.1099 & C19 & 0.0643 & C33 & 0.1168 \\
\hline C6 & 0.1016 & C20 & 0.1464 & C34 & 0.1910 \\
\hline C7 & 0.0844 & C21 & 0.1167 & C35 & 0.0623 \\
\hline C8 & 0.0991 & C22 & 0.1389 & C36 & 0.0633 \\
\hline C9 & 0.1674 & $\mathrm{C} 23$ & 0.1606 & C37 & 0.1149 \\
\hline C10 & 0.0576 & C24 & 0.0626 & C38 & 0.2177 \\
\hline C11 & 0.1078 & C25 & 0.1229 & C39 & 0.0450 \\
\hline C12 & 0.1290 & C26 & 0.1760 & C40 & 0.0965 \\
\hline C13 & 0.0886 & C27 & 0.1101 & C41 & 0.1457 \\
\hline C14 & 0.1251 & $\mathrm{C} 28$ & 0.0788 & $\mathrm{C} 42$ & 0.1929 \\
\hline
\end{tabular}

\section{Conclusions}

Due to the high technology industry contains many fields; in the actual development level of evaluation is not uniformity. As a result, there is still no recognized evaluation method. The set of evaluation system will key national high-tech zone as evaluation objects. At the same time, on the basis of the existing research, in the design of evaluation index system is mainly introduced the categories of "collaborative innovation" index, effective to evaluate high-tech zone collaborative innovation capability. Through research and analysis, evaluation index system of high-tech zone function in collaborative innovation in the index weight is as high as 0.2968 , therefore, we can conclude that the collaborative innovation plays an increasingly important role in the high-tech development evaluating the function of hi-tech zone in the perspective of collaborative innovation is very necessary, also it opens a new train of thought for the future study of high-tech zone function. (Graduate Innovation Fund of Sichuan Science and Engineering University Fund Code y20130 $01 ; "$ The interaction mechanism study of Regional Innovation System and High-tech Development Zone," Sichuan Science and Technology Hall Project (2013ZR0125); Science and technology proje cts of Zigong City, "The study of new industrialization and scientific and technological support cap abilities for Zigong City " (2012R08)

\section{References}

[1] Rogers, Larsen. Silicon Valley hot [M] .Economic Science Press, 1985

[2]Makecki EJ. The R\&D Location Decision of the Firm and Creative regions-A Survey [J].Technovation, 1987, (6)

[3]Malechi E.J., Nijkamp P. Technology and regional development: Some thoughts and policy [J].Environment and Planning C: Government and Policy, 1988, 6(4):383-399.

[4]Mei ShuE, Zhong Junwei, Hu Yidong. Evaluation index system of high-tech zones development level based on "second pioneering" [J]. Technological and economic, 2004, 04:15 -19 +29

[5]Chen Jiaxiang. Evaluation and measurement of national hi-tech zone function alienation deviation [J]. Technology Progress and Policy, 2009, 20:134-138. 
[6]Hu Shuhua, xie Jialong, Wang Song, Wang Jiao. Evaluation of high-tech zones Competitiveness based on method of multilevel fuzzy comprehensive [J] Based on Soft Science, 2011, 05:53-56.

[7]Zhang Gang, Chen Jin, Xu Qingrui. Study on collaborative innovation model based on technology, organization and culture [J]. Scientific Research, 1997 (2):56-61

[8]Zheng Gang, Zhu Ling, Jin Jun. Comprehensive and collaborative innovation: a five-stage comprehensive collaborative process model - a case study based Haier Group [J].Management Engineering, 2008 (2):24-30.

[9]Fan Decheng, Tang Xiaoxu. Performance Evaluation of Industry - University - Research Cooperative Technological Innovation Based on Fuzzy Integral[C].International Conference on Management Science\&Engineering,2009:1789-1795

[10]Jin Minghua, Zhang Xue. Analysis and Assessment on Risks of Enterprise-Customer Collaborative Innovation[C].International Conference on Management and Service Science, 2009:1-4

[11]Ou Guangjun, Liu Siyun, Jiang Huanyun, Cheng Houjin. Evaluation and empirical research on tech zone collaborative innovation capability under clusters perspective [J]. Technology Progress and Policy, 2013, 07:123-129. 